\title{
PID Controller design for multiple time delays system
}

\author{
Asma Karoui, Rihem Farkh, Moufida Ksouri \\ Laboratoire d'Analyse, Conception et Commande des Systèmes \\ Université de Tunis El Manar \\ Ecole Nationale d'Ingénieurs de Tunis \\ BP 37, Le Belvedère 1002, Tunis, Tunisia \\ asma.karoui@gmail.com, rihemfarkh@yahoo.fr, moufida ksouri@yahoo.fr
}

Received: August 5, 2019. Revised: August 22, 2021. Accepted: September 27, 2021. Published: November 27, 2021.

\begin{abstract}
This paper presents an approach of stabilization and control of time invariant linear system of an arbitrary order that include several time delays. In this work, the stability is ensured by PI, PD and PID controller. The method is analytical and needs the knowledge of transfer function parameters of the plant. It permits to find stability region by the determination of $K_{p}$, $K_{i}$ and $K_{d}$ gains.
\end{abstract}

\section{Keywords — PID Controller, multiple delay systems, stability region}

\section{INTRODUCTION}

Time delay systems are often encountered in various engineering systems such as electrical and communication network, chemical process, turbojet engine, nuclear reactor, hydraulic system; it is frequently a source of instability, oscillation and poor performance in many dynamic systems. Furthermore, delay makes system analysis and control design much more complex [7], [17].

The PID controller is widely applied in control engineering applications for many industries. The choice of the PID controller parameters leads to obtain a closed loop stable system.

Many researches have been applied the PID controller to different classes of dynamical systems [1]. Among these the particular class of time delay system has been investigated by means of several methods [8], [14], [15], [16], of which the Nyquist criterion, a generalization of the Hermite-Biehler Theorem, and the root location method. The main objective to design the PID controller is to ensure closed loop stability. Indeed, by using the Hermit-Biehler theorem applicable to the quasi-polynomials [9], [10], [11], a characterization of all values of the PI/PID stabilization gains for stable first order delay system is addressed. However, these results are not applicable to the second order delay system. In [2], [3], the stabilizing problem of PI/PID controller for second order delay system is analysed and then used to obtain all PI and PID gains that stabilize an interval first and second order delay system [4], [5].

The design methods of PID controllers can be analytically determined in the case of knowledge of the transfer function parameters or numerically in the case of the knowledge of the delay system frequency response [6], [12], [13].

Since these methods have been developed mainly for the case of a single system delay, the contribution of our work concerns the stabilization of system with several time delays by using the PI, PD and PID controllers. The proposed approach is based on the extension of the analytical method developed in [6], [12], [13].

The considered feedback structure is depicted in Fig. 1 and the related transfer functions of the process $G(s)$ and the controller $C(s)$ are given by:

$$
\begin{gathered}
G(s)=\sum_{i=1}^{N} G_{i}(s) e^{-\tau_{i} s} \\
C(s)=K_{p}+\frac{K_{i}}{s}+K_{d} s
\end{gathered}
$$

where $\mathrm{N}$ is the number of delays, $\tau_{i}$ is the time delay and $G_{i}$ is a continuous linear system of any order, $K_{p}, K_{i}$ and $K_{d}$ are the PID parameters.

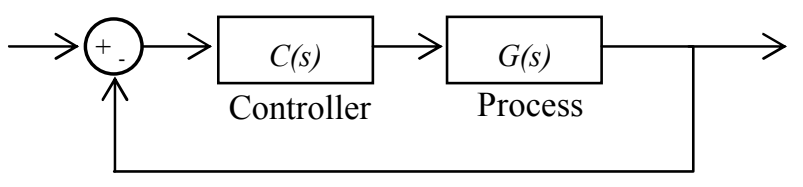

Figure 1: The closed-loop system with controller

The region of stability is found in the $\left(K_{p}, K_{i}\right)$ plane for PI controller, $\left(K_{p}, K_{d}\right)$ plane for PD controller and ( $K_{p}, K_{i}, K_{d}$ ) space for PID controller. This approach has been applied to continuous time linear systems of any order, with multiple time delays.

The paper is organized as follows: In part II stabilization approach with PI controller is presented, similarly 
stabilization approach with PD controller is developed in section III. The case of PID controller is discussed in section IV. Finally, simulation results are given in section V.

\section{STABILIZATION SEVERAL TIME DELAYS SYSTEM USING PI \\ CONTROLLER}

The considered plant is a continuous linear time-invariant system of any order that contains several time delays. It is described by its transfer function given by (1).

In this section, the stabilization of the plant is assured by the PI controller designed as follows:

$$
C(s)=K_{p}+\frac{K_{i}}{s}=\frac{K_{p} s+K_{i}}{s}
$$

The proposed method leads to an efficient calculation of the proportional and integral gains $K_{p}$ and $K_{i}$ achieving stability.

Let's note $\Delta(\mathrm{s})$ the closed loop characteristic polynomial of the process shown in Fig. 1.

In frequency domain, the characteristic polynomial is defined by:

$$
\Delta(j \omega)=1+C(j \omega) G(j \omega)=R_{\Delta}(\omega)+j I_{\Delta}(\omega)
$$

the transfer function can be then written as:

$$
\begin{aligned}
G(j \omega) & =\sum_{i=1}^{N} R_{i}(\omega)+j I_{i}(\omega) \\
& =\sum_{i=1}^{N} R_{i}(\omega)+j \sum_{i=1}^{N} I_{i}(\omega)
\end{aligned}
$$

where $R_{\Delta}$ and $I_{\Delta}$ are the real and the imaginary parts of the characteristic polynomial, respectively. $R_{i}$ and $I_{i}$ are the real and the imaginary parts of the transfer function $G_{i}(j \omega)$.

The stability region is determined when $\Delta(j \omega)$ is equal to zero:

$\Delta(j \omega)=1+\left(K_{p}-j \frac{K_{i}}{\omega}\right)\left(\sum_{i=1}^{N} R_{i}(\omega)+j \sum_{i=1}^{N} I_{i}(\omega)\right)=R_{\Delta}(\omega)+j I_{\Delta}(\omega)=0$

According to equations (4) and (5), the following results are obtained:

$$
\left\{\begin{array}{l}
R_{\Delta}(\omega)=1+K_{p} \sum_{i=1}^{N} R_{i}(\omega)+\frac{K_{i}}{\omega} \sum_{i=1}^{N} I_{i}(\omega) \\
I_{\Delta}(\omega)=K_{p} \sum_{i=1}^{N} I_{i}(\omega)-\frac{K_{i}}{\omega} \sum_{i=1}^{N} R_{i}(\omega)
\end{array}\right.
$$

Applying the real part and the imaginary part equal to zero leads to the following equations:

$$
\left\{\begin{array}{l}
K_{p} \sum_{i=1}^{N} R_{i}(\omega)+\frac{K_{i}}{\omega} \sum_{i=1}^{N} I_{i}(\omega)=-1 \\
K_{p} \sum_{i=1}^{N} I_{i}(\omega)-\frac{K_{i}}{\omega} \sum_{i=1}^{N} R_{i}(\omega)=0
\end{array}\right.
$$

Similar,

$$
\left\{\begin{array}{l}
\omega K_{p} \sum_{i=1}^{N} R_{i}(\omega)+K_{i} \sum_{i=1}^{N} I_{i}(\omega)=-\omega \\
\omega K_{p} \sum_{i=1}^{N} I_{i}(\omega)-K_{i} \sum_{i=1}^{N} R_{i}(\omega)=0
\end{array}\right.
$$

The $K_{p}$ and $K_{i}$ parameters are determined by solving the following system to ensure closed loop stability

$$
\left[\begin{array}{cc}
\omega \sum_{i=1}^{N} R_{i}(\omega) & \sum_{i=1}^{N} I_{i}(\omega) \\
\omega \sum_{i=1}^{N} I_{i}(\omega) & -\sum_{i=1}^{N} R_{i}(\omega)
\end{array}\right]\left[\begin{array}{l}
K_{p} \\
K_{i}
\end{array}\right]=\left[\begin{array}{l}
-\omega \\
0
\end{array}\right]
$$

The obtained expressions of $K_{p}$ and $K_{i}$ are:

where

$$
\left\{\begin{array}{c}
K_{p}(\omega)=-\frac{\sum_{i=1}^{N} R_{i}(\omega)}{|G(j \omega)|^{2}} \\
K_{i}(\omega)=-\omega \frac{\sum_{i=1}^{N} I_{i}(\omega)}{|G(j \omega)|^{2}}
\end{array}\right.
$$

\section{STABILIZATION SEVERAL TIME DELAYS SYSTEM USING PD CONTROLLER}

In this section, the same plant (1) is stabilized with a PD controller as shown in Fig. 1.

The transfer function of this controller is given by:

$$
C(s)=K_{p}+K_{d} s
$$

To obtain stability region in terms of proportional and derivative gains $K_{p}$ and $K_{d}$, the previous approach is applied in the case of several time delay system with PD controller

The closed loop characteristic polynomial $\Delta(s)$ is written as:

$$
\Delta(j \omega)=1+\left(K_{p}+j K_{d} \omega\right)\left(\sum_{i=1}^{N} R_{i}(\omega)+j \sum_{i=1}^{N} I_{i}(\omega)\right)=R_{\Delta}(\omega)+j I_{\Delta}(\omega)(14)
$$

by setting $\Delta(j \omega)$ equal to zero:

$$
\Delta(j \omega)=R_{\Delta}(\omega)+j I_{\Delta}(\omega)=0
$$

where: 


$$
\left\{\begin{array}{l}
R_{\Delta}(\omega)=1+K_{p} \sum_{i=1}^{N} R_{i}(\omega)-K_{d} \omega \sum_{i=1}^{N} I_{i}(\omega) \\
I_{\Delta}(\omega)=K_{p} \sum_{i=1}^{N} I_{i}(\omega)+K_{d} \omega \sum_{i=1}^{N} R_{i}(\omega)
\end{array}\right.
$$

the real part and the imaginary part equal to zero lead to the following equations:

$$
\left\{\begin{array}{l}
K_{p} \sum_{i=1}^{N} R_{i}(\omega)-K_{d} \omega \sum_{i=1}^{N} I_{i}(\omega)=-1 \\
K_{p} \sum_{i=1}^{N} I_{i}(\omega)+K_{d} \omega \sum_{i=1}^{N} R_{i}(\omega)=0
\end{array}\right.
$$

Equivalently the system can be written as:

$$
\left[\begin{array}{cc}
\sum_{i=1}^{N} R_{i}(\omega) & -\omega \sum_{i=1}^{N} I_{i}(\omega) \\
\sum_{i=1}^{N} I_{i}(\omega) & \omega \sum_{i=1}^{N} R_{i}(\omega)
\end{array}\right]\left[\begin{array}{l}
K_{p} \\
K_{d}
\end{array}\right]=\left[\begin{array}{l}
-1 \\
0
\end{array}\right]
$$

Solving (18), the results are as follows:

$$
\left\{\begin{array}{l}
K_{p}(\omega)=-\frac{\sum_{i=1}^{N} R_{i}(\omega)}{|G(j \omega)|^{2}} \\
K_{d}(\omega)=\frac{\sum_{i=1}^{N} I_{i}(\omega)}{\omega|G(j \omega)|^{2}}
\end{array}\right.
$$

where $|G(j \omega)|^{2}$ is given by (12).

\section{STABILIZATION SEVERAL TIME DELAYS SYSTEM USING PID CONTROLLER}

Considering the same system (1) shown in Fig. 1, we attempt to achieve stabilization with PID controller presented by:

$$
C(s)=K_{p}+\frac{K_{i}}{s}+K_{d} s=\frac{K_{p} s+K_{i}+K_{d} s^{2}}{s}
$$

The same approach is applied in the case of several time delay system with PID controller.

The closed loop characteristic polynomial $\Delta(\mathrm{s})$ is written as:

$$
\Delta(j \omega)=1-\left(\frac{-K_{p} \omega+j\left(K_{i}-K_{d} \omega^{2}\right)}{\omega}\right)\left(\sum_{i=1}^{N} R_{i}(\omega)+j \sum_{i=1}^{N} I_{i}(\omega)\right)=R_{\Delta}(\omega)+j I_{\Delta}(\omega)
$$

The stabilization region is determined by setting $\Delta(j \omega)$ to zero:

$$
\Delta(j \omega)=R_{\Delta}(\omega)+j I_{\Delta}(\omega)=0
$$

where:

$$
\left\{\begin{array}{l}
R_{\Delta}(\omega)=\omega+K_{p} \omega \sum_{i=1}^{N} R_{i}(\omega)-K_{d} \omega^{2} \sum_{i=1}^{N} I_{i}(\omega)+K_{i} \sum_{i=1}^{N} I_{i}(\omega) \\
I_{\Delta}(\omega)=K_{p} \omega \sum_{i=1}^{N} I_{i}(\omega)+K_{d} \omega^{2} \sum_{i=1}^{N} R_{i}(\omega)-K_{i} \sum_{i=1}^{N} R_{i}(\omega)
\end{array}\right.
$$

Real part and the imaginary part are setting to zero to obtain equation system of three unknown variable

$$
\left\{\begin{array}{l}
K_{p} \omega \sum_{i=1}^{N} R_{i}(\omega)+K_{i} \sum_{i=1}^{N} I_{i}(\omega)=-\omega+K_{d} \omega^{2} \sum_{i=1}^{N} I_{i}(\omega) \\
K_{p} \omega \sum_{i=1}^{N} I_{i}(\omega)-K_{i} \sum_{i=1}^{N} R_{i}(\omega)=-K_{d} \omega^{2} \sum_{i=1}^{N} I_{i}(\omega)
\end{array}\right.
$$

In the first step, the $K_{d}$ parameter is fixed. The $\left(K_{p}, K_{i}\right)$ plane is then determined by solving the following system:

$$
\left[\begin{array}{cc}
\omega \sum_{i=1}^{N} R_{i}(\omega) & \sum_{i=1}^{N} I_{i}(\omega) \\
\omega \sum_{i=1}^{N} I_{i}(\omega) & -\sum_{i=1}^{N} R_{i}(\omega)
\end{array}\right]\left[\begin{array}{l}
K_{p} \\
K_{i}
\end{array}\right]=\left[\begin{array}{l}
-\omega+K_{d} \omega^{2} \sum_{i=1}^{N} I_{i}(\omega) \\
-K_{d} \omega^{2} \sum_{i=1}^{N} R_{i}(\omega)
\end{array}\right]
$$

leading to the $K_{p}$ and $K_{i}$ expressions:

$$
\left\{\begin{array}{c}
K_{p}(\omega)=-\frac{\sum_{i=1}^{N} R_{i}(\omega)}{|G(j \omega)|^{2}} \\
K_{i}(\omega)=\omega^{2} K_{d}-\omega \frac{\sum_{i=1}^{N} I_{i}(\omega)}{|G(j \omega)|^{2}}
\end{array}\right.
$$

where $|G(j \omega)|^{2}$ is given by (12)

In the second step, the $K_{i}$ parameter is now fixed. The ( $K_{p}, K_{d}$ ) plane is then determined by solving the following system:

$$
\left[\begin{array}{cc}
-\omega \sum_{i=1}^{N} R_{i}(\omega) & \omega^{2} \sum_{i=1}^{N} I_{i}(\omega) \\
\omega \sum_{i=1}^{N} I_{i}(\omega) & \omega^{2} \sum_{i=1}^{N} R_{i}(\omega)
\end{array}\right]\left[\begin{array}{l}
K_{p} \\
K_{d}
\end{array}\right]=\left[\begin{array}{l}
\omega+K_{i} \sum_{i=1}^{N} I_{i}(\omega) \\
K_{i} \sum_{i=1}^{N} R_{i}(\omega)
\end{array}\right]
$$

$K_{p}$ is given by the same equations (26) and:

$$
K_{d}(\omega)=\frac{K_{i}}{\omega^{2}}+\frac{\sum_{i=1}^{N} I_{i}(\omega)}{\omega|G(j \omega)|^{2}}
$$

\section{Simulation RESUlts}

The proposed approach is illustrated on a linear system defined by two parallel subsystems, having two different time delays. The first subsystem is of order one and the second is of order three given by:

$$
G(s)=G_{1}(s) e^{-\tau_{1} s}+G_{2}(s) e^{-\tau_{2} s}
$$

where

$$
G_{1}(s)=\frac{K_{1}}{1+a_{1} s}, G_{2}(s)=\frac{K_{2}+K_{3} s}{1+b_{1} s+b_{2} s^{2}+b_{3} s^{3}}
$$


We suppose that: $\quad\left\{\begin{array}{l}K_{1}=0.5 \\ a_{1}=2 \\ \tau_{1}=1.5\end{array}\right.$ and $\left\{\begin{array}{l}K_{2}=1 \\ K_{3}=-0.5 \\ b_{1}=1 \\ b_{2}=3 \\ b_{3}=2 \\ \tau_{2}=0.6\end{array}\right.$

noting:

$G(j \omega)=G_{1}(j \omega)\left[\cos \left(\tau_{1} \omega\right)-j \sin \left(\tau_{1} \omega\right)\right]+G_{2}(j \omega)\left[\cos \left(\tau_{2} \omega\right)-j \sin \left(\tau_{2} \omega\right)\right]$

Developing (31), the results are as follows:

where

$$
\left\{\begin{array}{l}
R_{1}(\omega)=\frac{1}{1+A_{1}(\omega)^{2}}\left[K_{1} \cos \left(\tau_{1} \omega\right)-K_{1} A_{1}(\omega) \sin \left(\tau_{1} \omega\right)\right] \\
I_{1}(\omega)=-\frac{1}{1+A_{1}(\omega)^{2}}\left[K_{1} \sin \left(\tau_{1} \omega\right)+K_{1} A_{1}(\omega) \cos \left(\tau_{1} \omega\right)\right]
\end{array}\right.
$$

$$
A_{1}(\omega)=a_{1} \omega
$$

and

$$
\left\{\begin{array}{l}
R_{2}(\omega)=\frac{1}{B_{2}(\omega)^{2}+B_{1}(\omega)^{2}}\left[X_{1}(\omega) \cos \left(\tau_{2} w\right)+X_{2}(\omega) \sin \left(\tau_{2} w\right)\right] \\
I_{2}(\omega)=\frac{1}{B_{2}(\omega)^{2}+B_{1}(\omega)^{2}}\left[X_{2}(\omega) \cos \left(\tau_{2} w\right)-X_{2}(\omega) \sin \left(\tau_{2} w\right)\right]
\end{array}\right.
$$

where

$$
\left\{\begin{array}{l}
B_{1}(\omega)=b_{1} \omega-b_{3} \omega^{3} \\
B_{2}(\omega)=1-b_{2} \omega^{2} \\
X_{1}(\omega)=K_{2} B_{2}(\omega)+\omega K_{3} B_{1}(\omega) \\
X_{2}(\omega)=\omega K_{3} B_{2}(\omega)-K_{2} B_{1}(\omega)
\end{array}\right.
$$

$R_{1}, I_{1}$ are the real and imaginary parts of $G_{1}$, respectively $R_{2}, I_{2}$ are the real and imaginary parts of $G_{2}$, respectively

\section{A. Stabilization with PI controller}

$K_{p}$ and $K_{i}$ parameters are given by:

$$
\left\{\begin{array}{l}
K_{p}(\omega)=-\frac{R_{1}(\omega)+R_{2}(\omega)}{\left(R_{1}(\omega)+R_{2}(\omega)\right)^{2}+\left(I_{1}(\omega)+I_{2}(\omega)\right)^{2}} \\
K_{i}(\omega)=-\omega \frac{I_{1}(\omega)+I_{2}(\omega)}{\left(R_{1}(\omega)+R_{2}(\omega)\right)^{2}+\left(I_{1}(\omega)+I_{2}(\omega)\right)^{2}}
\end{array}\right.
$$

Substituting (32) and (33) into (34) leads to determine stability region in $\left(K_{p}, K_{i}\right)$ plane shown in the following figure:

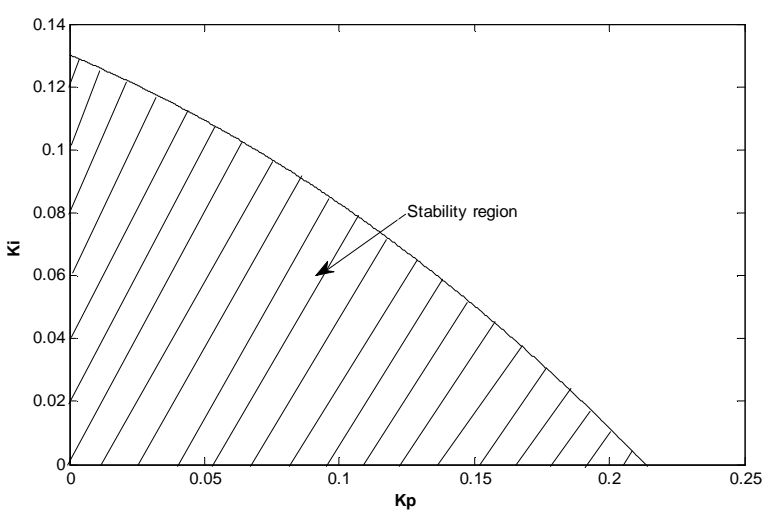

Figure 2: Stability region with PI Controller

In $\left(K_{p}, K_{i}\right)$ plane, the curve described by values of $K_{p}$ and $K_{i}$ given by equations (34) bounds a zone that represents stability region, which is displayed as shaded in Fig. 2.

Closed loop responses of this system are represented by the following figures:

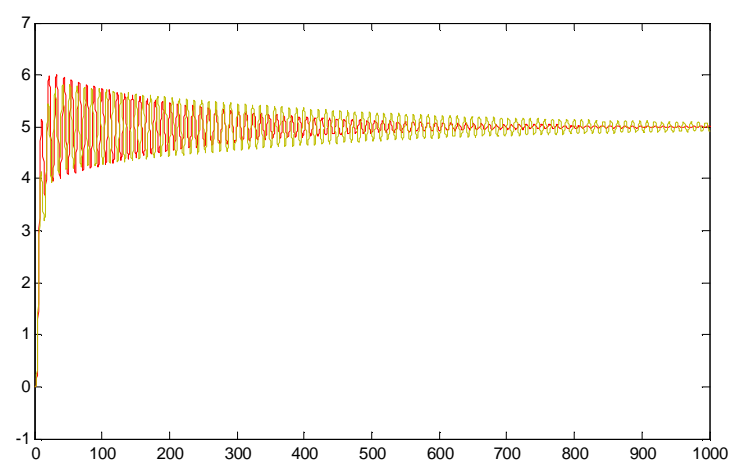

Figure 3: Closed loop step responses with PI Controller $(\mathrm{Kp}=0.05$, $\mathrm{Ki}=0.1$ : red response; $\mathrm{Kp}=0.1, \mathrm{Ki}=0.077$ : green response): stable case

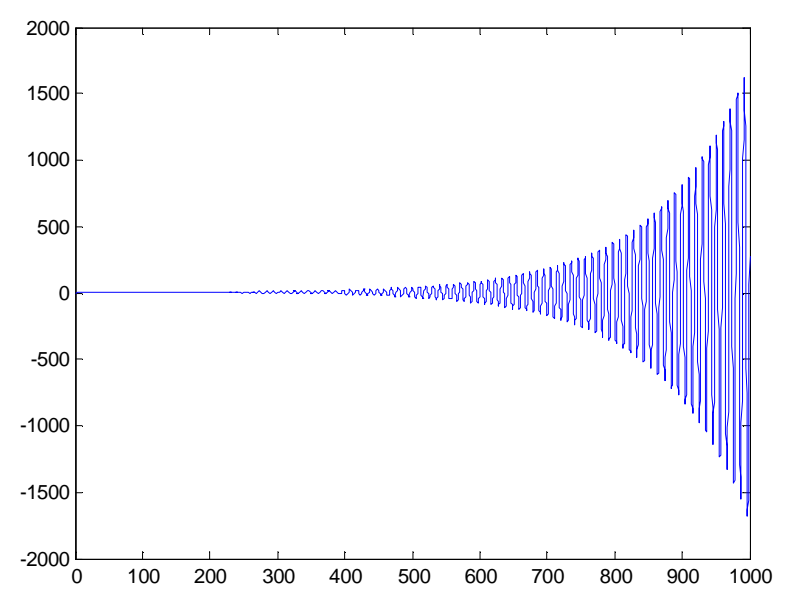

Figure 4: Closed loop step response with PI Controller $(\mathrm{Kp}=0.2, \mathrm{Ki}=0.04)$ : unstable case 
The parameters of the PI controller are altered according to their belonging to stability region.

Starting with $K_{p}=0.05, K_{i}=0.1$, the closed loop response system is described by the red response shown in Fig. 3.

Corresponding response for these gains displays that the system is stable in the shaded zone.

For $K_{p}=0.1, K_{i}=0.077$, these proportional and integral gains define a point belonging to the border of the shaded zone. Corresponding closed loop behavior system is shown by the green response in Fig. 3. In the transient regime, the system presents oscillations and it is stabilized in the steady state which represents the limit of the stability.

Finally, $K_{p}$ and $K_{i}$ parameters are chosen so that they define a point out of the region of stability. For $K_{p}=0.2$, $K_{i}=0.04$, the closed loop response system is presented in Fig. 4. The result shows that in the not shaded region, the system becomes unstable.

\section{B. Stabilization with PD controller}

The same approach is applied in the case of stabilization with PD controller.

The $K_{p}$ and $K_{d}$ expressions are given by:

$$
\left\{\begin{array}{l}
K_{p}(\omega)=-\frac{R_{1}(\omega)+R_{2}(\omega)}{\left(R_{1}(\omega)+R_{2}(\omega)\right)^{2}+\left(I_{1}(\omega)+I_{2}(\omega)\right)^{2}} \\
K_{d}(\omega)=\frac{I_{1}(\omega)+I_{2}(\omega)}{\omega\left(\left(R_{1}(\omega)+R_{2}(\omega)\right)^{2}+\left(I_{1}(\omega)+I_{2}(\omega)\right)^{2}\right)}
\end{array}\right.
$$

Substituting (32) and (33) into (35) leads to obtain $K_{p}$ and $K_{d}$ values.

As seen previously, the stability region which is shaded in Fig. 5 is bounded by the curve defined in $\left(K_{p}, K_{d}\right)$ plane.

Closed loop responses of this system for different $K_{p}$ and $K_{d}$ values are represented by the figures Fig. 6, Fig. 7.

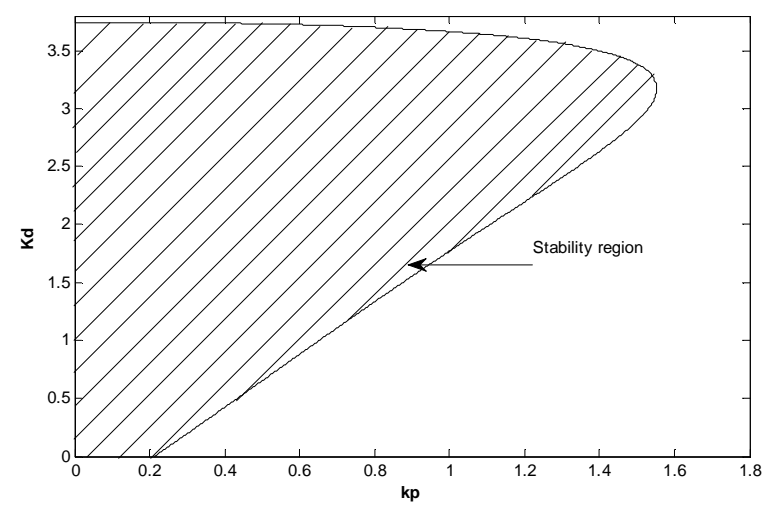




$$
\left\{\begin{array}{l}
K_{p}(\omega)=-\frac{R_{1}(\omega)+R_{2}(\omega)}{\left(R_{1}(\omega)+R_{2}(\omega)\right)^{2}+\left(I_{1}(\omega)+I_{2}(\omega)\right)^{2}} \\
K_{i}(\omega)=\omega^{2} K_{d}-\omega \frac{I_{1}(\omega)+I_{2}(\omega)}{\left(R_{1}(\omega)+R_{2}(\omega)\right)^{2}+\left(I_{1}(\omega)+I_{2}(\omega)\right)^{2}}
\end{array}\right.
$$

In the second step, the stability region can be determined in the $\left(K_{p}, K_{d}\right)$ plane with fixed $K_{i}$. The results obtained are as follow:

$$
\left\{\begin{array}{l}
K_{p}(\omega)=-\frac{R_{1}(\omega)+R_{2}(\omega)}{\left(R_{1}(\omega)+R_{2}(\omega)\right)^{2}+\left(I_{1}(\omega)+I_{2}(\omega)\right)^{2}} \\
K_{d}(\omega)=\frac{K_{i}}{\omega^{2}}+\frac{I_{1}(\omega)+I_{2}(\omega)}{\omega\left(\left(R_{1}(\omega)+R_{2}(\omega)\right)^{2}+\left(I_{1}(\omega)+I_{2}(\omega)\right)^{2}\right)}
\end{array}\right.
$$

Substituting (32) and (33) into (36) and (37) leads to obtain $K_{p}, K_{i}$ and $K_{d}$ values.

For each value of $K_{i}$, a stability region is defined in the ( $K_{p}, K_{d}$ ) plane. A three dimensional curve is then obtained by varying $K_{i}$ as shown in Fig. 8 .

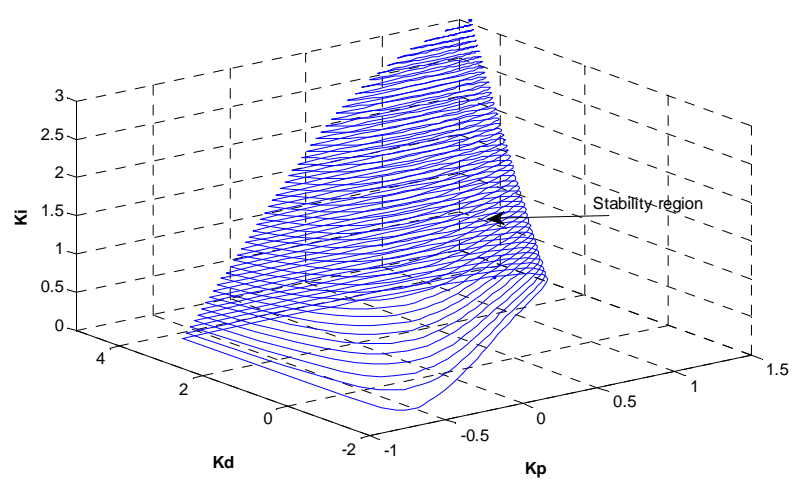

Figure 8: Stability region with PID Controller

The figures Fig. 9, Fig. 10 and Fig. 11 show the closed loop responses of the system for different chosen PID parameters.

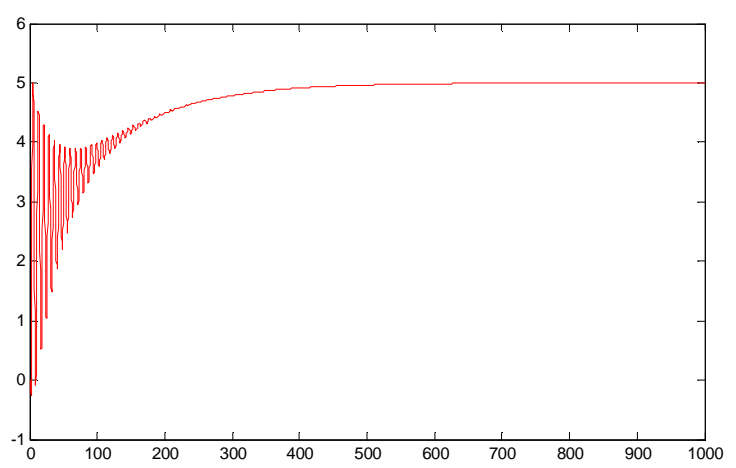

Figure 9: Closed loop step response with PID Controller $(\mathrm{Kp}=1, \mathrm{Ki}=2.085$, $\mathrm{Kd}=4.2$ ): stable case

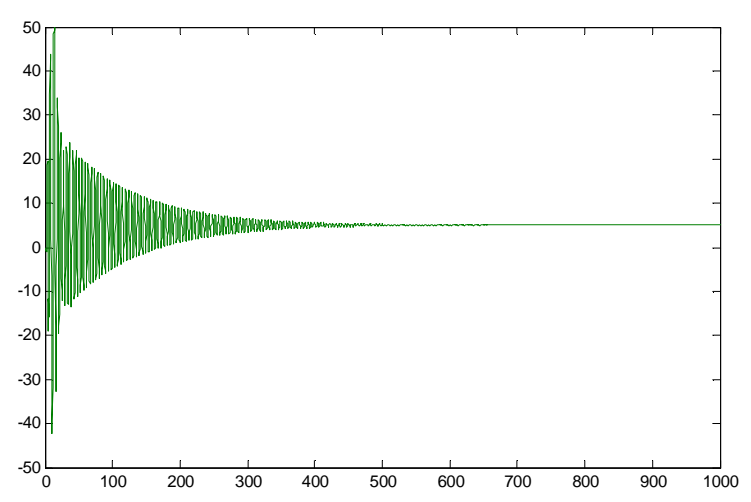

Figure 10: Closed loop step response with PID Controller $(\mathrm{Kp}=0.6, \mathrm{Ki}=2.085$, $\mathrm{Kd}=4.2$ ): stable case

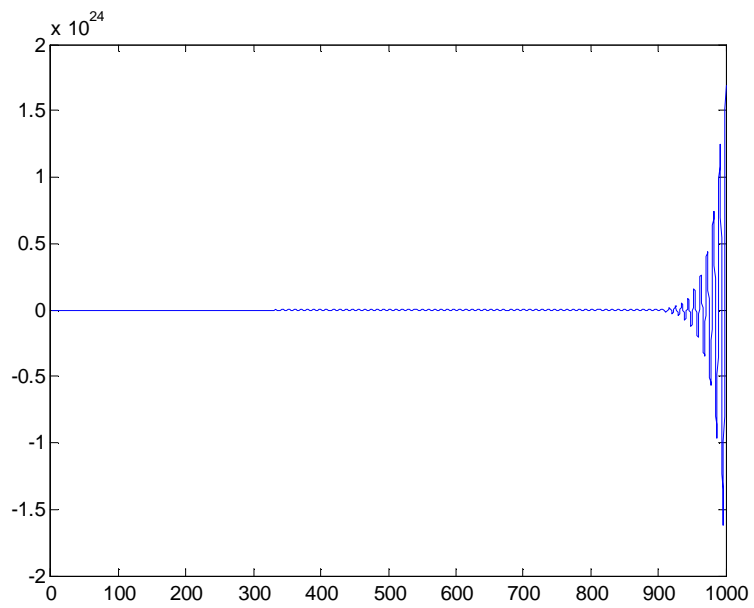

Figure 11: Closed loop step response with PID Controller $(\mathrm{Kp}=0.5, \mathrm{Ki}=0.05$, $\mathrm{Kd}=0.1$ ): unstable case

For $K_{p}=1, K_{i}=2.085$ and $K_{d}=4.2$, the closed loop system is stable.

In the case of $K_{p}=0.6, K_{i}=2.085$ and $K_{d}=4.2$, the system is on the limit of the stability in the closed loop.

Finally, for $K_{p}=0.5, K_{i}=0.05$ and $K_{d}=0.1$, the system is unstable in the closed loop.

\section{CONCLUSIONS}

The main contribution of this paper concerns the stabilization of continuous linear time invariant system of any order and which presents several delays using a PID controller. The proposed approach is based on mathematical calculation of the proportional, derivative and integral gains by extracting the real and imaginary parts of the system transfer function. This not complicated method leads to the determination of the stability regions in $\left(K_{p}, K_{i}\right)$ plane for PI controller, ( $K_{p}, K_{d}$ ) plane for PD controller and $\left(K_{p}, K_{i}, K_{d}\right)$ space for PID controller. 
The simulation results point out the correspondence between the time domain responses and the obtained stability regions. This proposed method is then efficient to stabilize system with any order and several time delays.

\section{REFERENCES}

[1] K. J. Astrom and T. Hagglund, PID controllers: Theory, design and tuning, Instrument Society of America, Research Triangle Park, NC, 1995.

[2] R. Farkh, K. Laabidi and M. Ksouri, "PI control for second order delay system with tuning parameter optimization", International Journal of Electrical, Computer and System Engineering, WASET 2009.

[3] R. Farkh, K. Laabidi and M. Ksouri, "Computation of all stabilizing PID gains for second order delay system", Mathematical Problem in Engineering, Vol. 2009, Article ID 212053, 17 pages, 2009. doi: $10.1155 / 2009 / 212053$.

[4] R. Farkh, K. Laabidi and M. Ksouri, "Robust stabilization for uncertain second order time-lag system", The Mediterranean Journal of Measurement and Control, Vol. 5, No. 4, 2009.

[5] R. Farkh, K. Laabidi and M. Ksouri, "Robust PI/PID controller for interval first order system with time delay", International Journal of Modelling Identification and Control, Vol. 13, No.1/2, pp. 67 - 77, 2011.

[6] T. Lee, J. Watkins, T. Emami, and S. Sujoldžić, "A unified approach for stabilization of arbitrary order continuous-time and discrete-time transfer functions with time delay using a PID controller", IEEE Conference on Decision and Control, New Orleans, LA, USA, Dec. 1214, 2007.

[7] S. I. Niculescu, Delay effects on stability, Springer, London, 2001.
[8] G. J. Silva, A. Datta and S. P. Bhattacharyya, PID controllers for timedelay systems. Boston: Birkhäuser, 2005.

[9] G. J. Silva, A. Datta and S. P. Bhattacharyya, "Stabilization of time delay system", Proceedings of the American Control Conference, pp. 963-970, 2000.

[10] G. J. Silva, A. Datta and S. P. Bhattacharyya., "PI stabilization of firstorder systems with time-delay", Automatica, Vol. 37, No.12, pp.20252031, 2001.

[11] G. J. Silva, A. Datta and S. P. Bhattacharyya, "Stabilization of firstorder systems with time delay using the PID controller", Proceedings of the American Control Conference, pp. 4650-4655, 2001.

[12] S. Sujoldzic and J. Watkins, "Stabilization of an arbitrary order transfer function with time delay using PI and PD controllers", Proceedings of the American Control Conference, pp. 2427-2432. Minneapolis, MN, June 2006.

[13] S. Sujoldžic and J. Watkins, "Stabilization of an arbitrary order transfer function with time delay using PID controllers", Proceedings of the 45th IEEE Conference on Decision \& Control, San Diego, CA, USA, December 13-15, 2006.

[14] N. Tan, "Computation of stabilizing PI and PID controllers for processes with time delay ", ISA Transactions, Vol. 44, pp. 213-223, 2005.

[15] S. Tavakoli and P. Fleming, "Optimal tuning of PI controllers for first order plus dead time/long dead time models using dimensional analysis", European Control Conference, UK, 2003.

[16] J. Watkins and G. Piper, "On the impact of cross-link delays on spacecraft formation control", Journal of the Astronautical Science, Vol. 53, NI, pp. 83-101, 2006.

[17] Q. C. Zhong, Robust control of time delay system, Springer, London, 2006.

\section{Creative Commons Attribution License 4.0 (Attribution 4.0 International, CC BY 4.0)}

This article is published under the terms of the Creative Commons Attribution License 4.0

https://creativecommons.org/licenses/by/4.0/deed.en_US 